ESAIM: PROCEEDINGS, September 2005, Vol.14, 72-88

Eric Cancès \& Jean-Frédéric Gerbeau, Editors

DOI: $10.1051 /$ proc:2005007

\title{
MATHEMATICAL MODELLING OF THE CARDIOVASCULAR SYSTEM AND SKELETAL MUSCLE INTERACTION DURING EXERCISE
}

\author{
Carlo D'Angelo ${ }^{1}$ and Yves Papelier ${ }^{2}$
}

\begin{abstract}
In this work we present a mathematical model for the coupling of cardiovascular system and metabolism under sport efforts. Effects such as oxygenation, heart rates, flow rates, blood pressure and mass transfer to tissues will be considered. A special attention will be payed to the modelling of the regulatory mechanisms (baroreflex and chemoreflex effectors) affecting the heart and the peripheral circulation. We aim at extending the existing models to describe metabolism, hemodynamics and mass transport during exercise, providing a framework to understand their mutual interactions. Numerical issues are presented for different workloads, and a quantitative analysis of dynamic aerobic exercise is discussed.
\end{abstract}

Résumé. Dans ce travail nous présentons un modèle mathématique de couplage du système cardiovasculaire au métabolisme lors d'efforts intenses de type sportif. Les conséquences au plan cardiaque, au plan des pressions et débits sanguins, de l'oxygénation, et des transferts de matière au sein des tissus sont prises en compte. Une attention spéciale est apportée à la modélisation des mécanismes cardiovasculaires de régulation mis en jeu (baroréflexe et chémoréflexe), d'un point de vue cardiaque ou vasculaire périphérique. Nous nous proposons d'élargir les modèles existants de métabolisme, de l'hémodynamique et des transferts de matière lors de l'exercice, apportant ainsi une architecture utile à la compréhension de leurs interactions mutuelles. Les résultats de simulations numériques sont présentés pour différents niveaux de charge d'exercice, et l'analyse quantitative de l'exercice dynamique aérobie est discutée.

\section{INTRODUCTION}

The analysis of the physiological response of the human body to the increased metabolic rate during exercise is a very complex subject. On one hand, there is a strong interaction between the cardiovascular system and the skeletal muscle metabolism; on the other, relatively few biochemical measurements and mathematical models are available to provide a general quantitative description of the coupling between hemodynamics and metabolism. Beyond doubt, the following aspects are of primary importance in understanding the physiological adaptations under effort conditions:

(1) the biochemical processes in skeletal muscle;

(2) the substrate (oxygen, glucose, lactate, ...) transfer between blood and tissues;

(3) the cardiovascular feedback with respect to the arterial pressure and substrate concentrations (i.e. baroreflex and chemoreflex effect).

Any mathematical model of an exercise protocol should include a suitable integration between these items. Very often the existing studies either focus on the cardiovascular part (as in [17]) or investigate the biochemical

1 carlo.dangelo@epfl.ch, EPFL/SB/IACS/CMCS, Ecole Polytechnique Fédérale de Lausanne (Switzerland).

2 yves.papelier@kb.u-psud.fr, Hôpital Antoine-Béclère, Clamart (France) and INRIA Rocquencourt, Le Chesnay (France).

(c) EDP Sciences, SMAI 2005 
processes (see [14], [12], [15]); an integrative approach is developed in [3], but the topic of the autoregulation of the cardiovascular system is absent. In the next sections, we first introduce the physiological framework of the problem and discuss the typical parameters (that will be the variables of our model) obtained from standard measurements, considering for instance an individual running on a treadmill or riding a cycloergometer; then we present our model, and finally we study the results of the numerical simulations, comparing it with available data when it is possible.

\subsection{Cardiovascular parameters and auto-regulation}

In a standard treadmill test, sport physicians usually measure some typical parameters related to gas exchange, like the oxygen uptake $\dot{\mathrm{V}}_{\mathrm{O}_{2}}$ and the carbon dioxide production $\dot{\mathrm{V}}_{\mathrm{CO}_{2}}$. Both $\dot{\mathrm{V}}_{\mathrm{O}_{2}}$ and $\dot{\mathrm{V}}_{\mathrm{CO}_{2}}$ are mass transfer rates (they are expressed for instance in $[\mathrm{L} / \mathrm{min}]$ ); values of these quantities are obtained by a digital system that samples the expired air, and measures chemical concentrations by mass spectrometry ${ }^{1}$.

Among the other parameters measured in such a test, there is the heart rate $H R$, whose units are beats per minute $[\mathrm{bpm}]=[1 / \mathrm{min}]$. The heart rate is related to the cardiac output $\dot{Q}$ and the stroke volume $\mathrm{V}_{\mathrm{s}}$ by the simple relation

$$
\dot{Q}=H R \mathrm{~V}_{\mathrm{s}},
$$

where $\dot{Q}$ (usually [L/min]) is the total volume of blood pumped by the heart through the circulatory system per unit time, and $\mathrm{V}_{\mathrm{s}}$ is the volume of blood ejected at each heart contraction.

A normal value for cardiac output at rest is close to 5-6 L/ min. This increases to approximately 15-25 L/ min in healthy adults at maximal exercise. Stroke volume ranges from approximately $80 \mathrm{~mL}$ during upright rest up to $130 \mathrm{~mL}$ during exercise. In elite endurance athletes however, stroke volumes can be as high as $180-200 \mathrm{~mL}$.

In dynamic exercise heart rate increases with respect to the workload and oxygen consumption [1]. Typically, heart rate ranges from $50-70 \mathrm{bpm}$ at rest up to $180-200 \mathrm{bpm}$ during maximal effort.

The measurement of substrate concentrations in blood is more difficult, but it is possible via catheter sampling. We denote the chemical concentrations by $\left[\mathrm{O}_{2}\right],\left[\mathrm{CO}_{2}\right]$, and so on: an important role in exercise physiology is played by the blood concentration of $\mathrm{O}_{2}$ in arteries, $\left[\mathrm{O}_{2}\right]_{a}$, and in veins, $\left[\mathrm{O}_{2}\right]_{v}$. The arterial-venous $\mathrm{O}_{2}$ difference

$$
\Delta_{v}^{a}\left[O_{2}\right]=\left[O_{2}\right]_{a}-\left[O_{2}\right]_{v}
$$

is an index of the oxygen consumption by tissues (especially skeletal muscle). Generally chemical concentrations in blood are expressed in terms of molar values $[\mathrm{mmol} / \mathrm{L}]$ or fractional values $[\mathrm{mL} / \mathrm{L}]$. The arteriovenous $\mathrm{O}_{2}$ difference is related to $\dot{\mathrm{V}}_{\mathrm{O}_{2}}$ and $\dot{Q}$ by

$$
\dot{\mathrm{V}}_{\mathrm{O}_{2}}=\dot{Q} \Delta_{v}^{a}\left[O_{2}\right]
$$

which express the balance between oxygen intake and outflow.

We point out that it is possible to measure chemical concentrations in tissues as well, after a local biopsy; however, this is a rather complex procedure, so that known in vivo data for human metabolism under effort conditions are very few. Often the units associated to the concentration in a tissue are $[\mathrm{mmol} / \mathrm{kgdw}]$, where [kgdw] means kg of dry weight of tissue. Recently, MRI is becoming a powerful tool for non-invasive measurement $[21]$.

In dynamic exercise whole body arteriovenous oxygen difference $\Delta_{v}^{a}\left[\mathrm{O}_{2}\right]$ increases with exercise intensity and oxygen consumption. Typically, $\Delta_{v}^{a}\left[\mathrm{O}_{2}\right]$ ranges from $50 \mathrm{~mL}\left(\mathrm{O}_{2}\right) / \mathrm{L}(\mathrm{blood})$ at rest to approximately $150 \mathrm{~mL} / \mathrm{L}$ during maximal exercise. Arterial oxygen content of approximately $200 \mathrm{~mL}\left(\mathrm{O}_{2}\right) / \mathrm{mL}$ (blood) does not change substantially with increasing exercise intensity but venous content falls considerably until approximately $85 \%$ of the oxygen is extracted at maximal effort. At rest only a small percentage of the total capillaries in a given

\footnotetext{
${ }^{1}$ Generally one measures the fractional concentrations $F_{e}$ of expired gases, and this provides the pulmonary gas exchange rates as the fractional concentrations of inspired gases $F_{i}$ are known. For example,

where $\dot{\mathrm{V}}_{e}$ is the ventilation.

$$
\dot{\mathrm{V}}_{\mathrm{O}_{2}}=\dot{\mathrm{V}}_{e}\left(F_{i, O_{2}} \frac{1-F_{e, O_{2}}-F_{e, C O_{2}}}{F_{i, N_{2}}}-F_{e, O_{2}}\right) \text {, }
$$
}


tissue are open. During dynamic exercise the reduced oxygen concentration causes the arterioles and capillaries to dilate, and the opening of closed capillaries: this effect, called chemoreflex, provide more blood (and then more oxygen) to the tissues. It is a biochemical effect which involves also the cardiovascular system, because it implies a dramatic fall in hydrodynamic resistance ${ }^{2}$ of the tissues fed by the circulatory system.

This high variability in the peripheral resistance of the small vessels influences the cardiac activity via the baroreflex effect, which is a physiological regulation system that maintains the arterial pressure in the large arteries close to a reference mean value (typically $90-100 \mathrm{mmHg}$ ). It consists of stretch receptors in most of the large arteries of the thorax and neck (especially the aortic arch and carotid sinus), which respond to changes in pressure and rate of change in pressures between 60 and $180 \mathrm{mmHg}$ for the carotids and 90 and $210 \mathrm{mmHg}$ in the aorta. The receptors act on the heart rate and on the heart contractility: for example, if the arterial pressure decreases, the firing rate of the aortic arch and carotid sinus increases, and this causes an increase in $H R$ and in heart contractility (that is, as we will see, in $\mathrm{V}_{\mathrm{s}}$ ). The baroreflex effector controls also the peripheral resistance of the arterioles and capillaries, but during exercise the main effect is on the heart. The baroreflex is a very important feedback system because the arterial pressure is a vital parameter (if the blood pressure does fall, the oxygen partial pressure does; conversely, high pressures are related to cardiovascular diseases).

Typically, the oxygen fall in exercising tissues causes (via chemoreflex) a reduction in their hydrodynamic resistance and hence in the overall systemic resistance; the baroreflex acts immediately to compensate the arterial pressure decrease. Hence, the mean arterial pressure $(M A P)$ does not change significantly (generally an increase up to $125-130 \mathrm{mmHg}$ is observed), while the cardiac output does (see Table 1 , where the systemic vascular resistance is calculated as $R=M A P / \dot{Q}$, see for instance [7]).

\begin{tabular}{|c|c|c|c|}
\hline Parameter & Rest & Exercise & $\Delta \%$ \\
\hline \hline Cardiac output $\dot{Q}[\mathrm{~L} / \mathrm{min}]$ & 6 & 21 & $+250 \%$ \\
\hline Mean Arterial Pressure $M A P[\mathrm{mmHg}]$ & 90 & 125 & $+40 \%$ \\
\hline Systemic resistance $R[\mathrm{mmHg} \min / \mathrm{L}]$ & 15 & 5 & $-66.7 \%$ \\
\hline
\end{tabular}

TABLE 1 . Differences in $\dot{Q}, M A P$ and $R=M A P / \dot{Q}$ between rest and strenuous dynamic exercise.

\begin{tabular}{|c|c|c||c|c|}
\hline Organ $i$ & $\dot{Q}_{i}(\mathrm{~mL} / \mathrm{min})$, rest & $\dot{Q}_{i} / \dot{Q} \%$ & $\dot{Q}_{i}(\mathrm{~mL} / \mathrm{min})$, exercise & $\dot{Q}_{i} / \dot{Q} \%$ \\
\hline \hline Brain & 650 & 13 & 750 & 4 \\
\hline Heart & 215 & 4 & 750 & 4 \\
\hline Skeletal Muscle & 1030 & 20 & 12500 & 73 \\
\hline Skin & 430 & 9 & 1900 & 11 \\
\hline Kidney & 950 & 20 & 600 & 3 \\
\hline Splanchnic Organs & 1200 & 24 & 600 & 3 \\
\hline Others & 545 & 10 & 400 & 2 \\
\hline \hline Total & 5000 & 100 & 17500 & 100 \\
\hline
\end{tabular}

TABLE 2. Flow rates at rest and at strenuous dynamic exercise [7] .

\footnotetext{
${ }^{2}$ This is due to the Poiseuille's law for a cylindric tube. If a pressure jump $\Delta P$ is applied between the inlet and the outlet, the flow rate is given by

$$
\dot{Q}=\frac{\Delta P}{R}, \quad R=\frac{8 \eta l}{\pi r^{4}},
$$

where $l$ is the tube length, $r$ the section radius, and $\eta$ the blood viscosity. $R$ is called haemodynamic resistance.
} 
To sustain physical activity for more than a few seconds requires dramatic circulatory adjustments to ensure adequate perfusion of the exercising skeletal muscle as well as of vital organs such as the heart and brain [8]: in fact, the demand of blood flow in a large volume of exercising muscle can approximate the maximal cardiac output [22]. Thus, the cardiovascular system has to control the delicate balance between pressure regulation and oxygen delivery, being the redistribution of the blood flow to each tissue affected by the local state of vessel dilatation and number of open capillaries (as reported in Table 2). The exact mechanisms involved in this integrated process are not entirely known. It is established that hemodynamic adaptations to exercise are mediated by a decrease in parasympathetic and an increase in sympathetic activity controlled both by feed-forward stimuli from increased central drive (due to the voluntary muscle contraction) and by feedback arising from mechanically and metabolically sensitive afferent nerve endings in the large arteries and contracting skeletal muscles.

\subsection{Gas exchange and ventilation}

Generally in a treadmill test a linearly increasing workload is imposed. In the time course of $\dot{\mathrm{V}}_{\mathrm{O}_{2}}=\dot{\mathrm{V}}_{\mathrm{O}_{2}}(t)$ for such a test, a maximal oxygen uptake $\dot{\mathrm{V}}_{\mathrm{O}_{2} \max }$ is observed. For young subjects, this plateau is usually 10 20 times the basal $\dot{\mathrm{V}}_{\mathrm{O}_{2}}$ uptake and cannot be exceeded, although it can be increased by appropriate training in high-fit up to 20 - 22 times the basal $\dot{\mathrm{V}}_{\mathrm{O}_{2}}$.

This maximal oxygen uptake is an useful but imperfect predictor of the ability to perform prolonged dynamic external work or, more specifically, of endurance athletic performance, because in this case the energy currency of muscles is the aerobic one (see the next section). In principle, as there are several physiological mechanisms controlling the ventilation, we should consider a model for the gas exchange; but for healthy people the limiting factor to aerobic performances is not represented by lungs (also at a very high cardiac output, the concentration gradient between the alveolar oxygen and the venous one is often high enough to saturate the arterial blood), but by the cardiovascular system. In fact, as described in Table 3, we can observe that the arterial partial

\begin{tabular}{|c|c|c|c|c|}
\hline Exercise intensity & $\dot{\mathrm{V}}_{e}(\mathrm{~L} / \mathrm{min})$ & $\mathrm{P}_{\mathrm{O}_{2}}(\mathrm{mmHg})$ & $\mathrm{P}_{\mathrm{CO}_{2}}(\mathrm{mmHg})$ & Blood $\mathrm{pH}$ \\
\hline \hline Rest & 5 & 103 & 36 & 7.40 \\
\hline Walking & 20 & 103 & 36 & 7.40 \\
\hline Jogging & 45 & 106 & 44 & 7.40 \\
\hline Running fast & $75-44$ & 110 & 44 & 7.32 \\
\hline
\end{tabular}

TABLE 3. Acute respiratory response to graded dynamic exercise in a 30-year-old untrained woman.

pressure $\mathrm{P}_{\mathrm{O}_{2}}$ and $\mathrm{P}_{\mathrm{CO}_{2}}$ are almost the same at rest and under effort conditions. Hence, in this work we do not consider a ventilatory model and we suppose that $\left[\mathrm{O}_{2}\right]_{a}$ and $\left[\mathrm{CO}_{2}\right]_{a}$ are given and constant.

In Table 3 a decrease in blood $\mathrm{pH}$ (acidosis) with respect to exercise intensity is observed. From the midway point between $\dot{\mathrm{V}}_{\mathrm{O}_{2} \text { rest }}$ and $\dot{\mathrm{V}}_{\mathrm{O}_{2} \text { max }}$, lactic acid begins to accumulate in blood. This point, that depends on the type of work involved and on the level of fitness of the subject, is called lactate threshold. Lactate concentration gradually rises with work intensity, as more and more muscle fibers must rely on anaerobic metabolism: this causes metabolic acidosis, which is partially responsible for muscle fatigue.

\subsection{Metabolism}

Several chemicals are involved in muscle contraction: a large number of reactions take place in each fiber in order to transform the chemical energy of certain molecular bounds in mechanical work. In particular, this is obtained from ATP (adenosine triphosphate) breakdown as follows:

$$
A T P \stackrel{\mathrm{MR}}{\longrightarrow} A D P+P i+\text { mechanical energy }(\simeq 30 \mathrm{KJ})+\text { heat }
$$


where $P i$ is an inorganic phosphate group, and $A D P$ is the molecule of adenosine diphosphate. We denote by MR the metabolic rate, that is the rate at which the $A T P$ is converted in $A D P$ in the skeletal muscle tissue (in $[\mathrm{mmol} / \mathrm{sec}]$ ). As muscle contractions rely completely on this reaction, MR is related to the power output during exercise (but as the amount of the mechanical energy available for muscular work obtained from ATP breakdown does depend on the exercise level [13], the relationship is generally nonlinear ).

Several reactions act in order to sustain the energy production in cells by restoring $A T P$ : as in [2], we will assume the following metabolic pathways in tissues (see [9] for a general survey on this approach).

(1) Phosphocreatine breakdown: this is the most important pathway for short-term ATP production. The reaction is

$$
P C+A D P \stackrel{P C b}{\longrightarrow} C R+A T P,
$$

where creatine $(C R)$ and $A T P$ are produced from phospocreatine $(P C)$ and $A D P$. We denote by $P C \mathrm{~b}$ this reaction. As source of $A T P$, the phosphagen system can only supply a cell for 8 to 10 seconds during the most strenuous exercise; then the $P C$ concentration becomes too small for sustaining the reaction.

(2) Phosphocreatine synthesis $(P C \mathrm{~s})$ : this is the inverse pathway of $P C \mathrm{~b}$, and allows to restore the $P C$ stores using ATP:

$$
C R+A T P \stackrel{P C \mathrm{~s}}{\longrightarrow} P C+A D P .
$$

(3) Anaerobic glycolysis. The overall chemical reaction is the following one (we denote it by GLb, or glucose breakdown):

$$
G L+2 A D P+2 N A D \stackrel{G L \mathrm{~b}}{\longrightarrow} 2 P Y+2 A T P+2 N A D H .
$$

Like most chemical reactions, glycolysis slows down as its products, pyruvic acid $P Y$ and the reduced coenzyme $N A D H$, build up.

(4) Pyruvate reduction. In order to speed up the glycolysis, pyruvate can be reduced and converted to lactate:

$$
P Y+N A D H \stackrel{P Y \mathrm{r}}{\longrightarrow} L A+N A D .
$$

Lactic acid itself eventually builds up, slowing metabolism and contributing to muscle fatigue.

(5) Gluconeogenesis. In splanchnic organs (namely in the liver) the pyruvate can be converted back to glucose:

$$
2 P Y+2 A T P+2 N A D H \stackrel{G L \mathrm{~s}}{\longrightarrow} G L+2 A D P+2 N A D .
$$

This pathway $(G L \mathrm{~s})$ is not observed in skeletal muscle.

(6) Pyruvate oxidation: this pathway allows to metabolize aerobically the pyruvic acid. Pyruvic acid is converted to a molecule called acetyl group and put into the Krebs cycle, from which high energy electrons are released to $N A D$ :

$$
P Y+5 N A D \stackrel{P Y o}{\longrightarrow} 5 \mathrm{NADH}+3 \mathrm{CO}_{2} .
$$

We denote this reaction by $P Y$ o (pyruvate oxidation).

(7) Oxidative phosphorylation. The high energy electrons obtained by the oxidation of $P Y$ are sent to a process within the mitochondria known as the electron transport chain which produces $A T P$, the waste products being $\mathrm{CO}_{2}$ and $\mathrm{H}_{2} \mathrm{O}$ :

$$
3 A D P+N A D H+\frac{1}{2} \mathrm{O}_{2} \stackrel{\mathrm{OP}}{\longrightarrow} 3 A T P+N A D+\mathrm{H}_{2} \mathrm{O} .
$$

This reaction will be denoted OP (oxidative phosphorylation). In this process, $\mathrm{O}_{2}$ is one of the reactants: this reaction is responsible for the high oxygen demand of tissues during exercise. 
(8) Glycogen synthesis and breakdown $(G Y \mathrm{~s}, G Y \mathrm{~b})$ : skeletal muscle and liver contain a certain store of glycogen $(G Y)$, a big molecule which is a sort of gathering of several glucose molecules. Glycogen can provide glucose by the following reaction:

$$
G Y+A D P \stackrel{G Y \mathrm{~b}}{\longrightarrow} m G L+A T P
$$

where $m$ is the number of $G L$ molecules in one $G Y$ molecule. However, $G Y$ can be restored from $G L$ via the following pathway

$$
m G L+A T P \stackrel{G Y \mathrm{~s}}{\longrightarrow} G Y+A D P .
$$

(9) Lactate oxidation $(L A \mathrm{o})$ : this is the inverse reaction of $P Y$ r. Pyruvate can be produced starting from lactate:

$$
L A+N A D \stackrel{L A \circ}{\longrightarrow} P Y+N A D H .
$$

(10) Free Fatty Acid oxidation $(F A o)$ : cells can oxidize free fatty acids $(F A)$ in order to provide $N A D H$ for the electron transport chain. The stoichiometry of this reaction is the following one:

$$
F A+43 N A D \stackrel{F A o}{\longrightarrow} 16 C O_{2}+43 N A D H
$$

In Figure 1 are depicted the main biochemical pathways we will consider. As pointed out in Figure 1, there

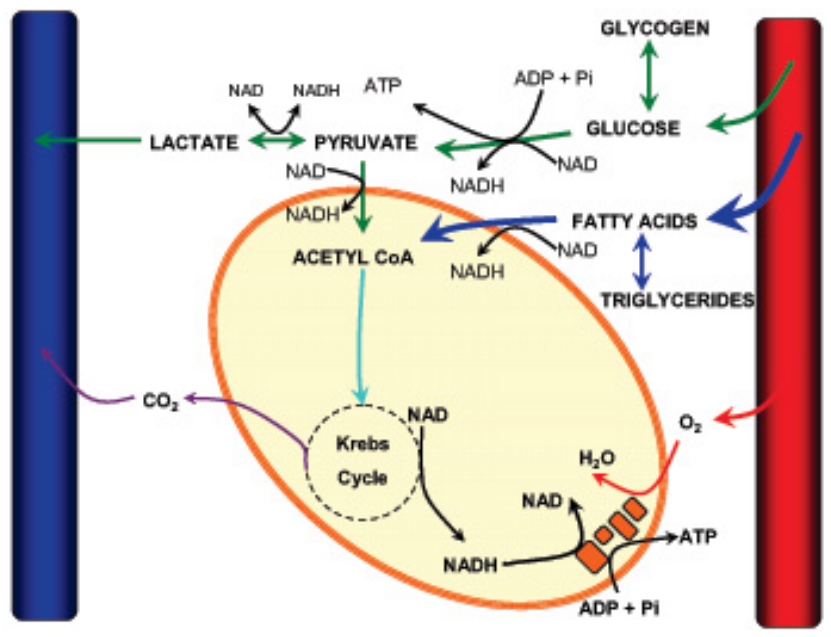

FiguRE 1. Sketch of the main metabolic pathways.

are some chemicals which are transported by the blood, and other chemicals which are not. We assume that $P C, C R, A D P, A T P, N A D, N A D H$ and $G Y$ are not transported; they are produced and consumed inside the tissue.

\section{Cardiovascular Model}

As we are not interested in the pulmonary system, we can consider a reduced cardiovascular model containing only the principal circulation (and not the pulmonary one). The model scheme is represented in Figure 2: the block $\mathrm{RH} / \mathrm{L} / \mathrm{LH}$ represents the right heart, the pulmonary circulation and the lungs, and the left heart. The left ventricle pumps the blood in the main arteries with a flow rate $\dot{Q}_{a}$ (corresponding to the cardiac output 


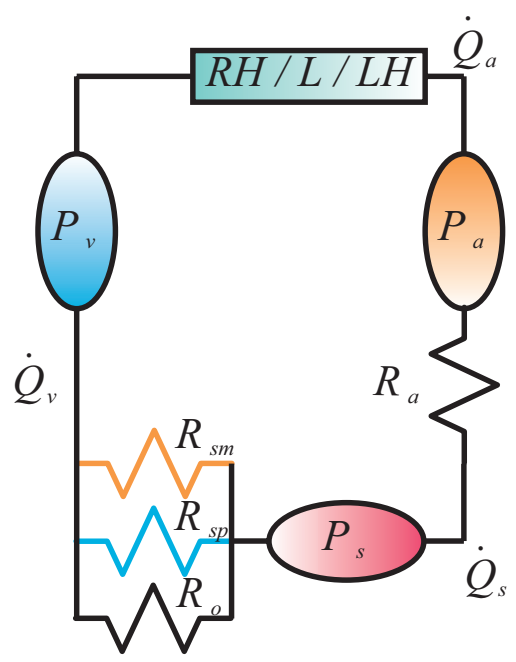

FIGURE 2. Scheme of a reduced circulatory system.

$\dot{Q}$ ). The main arteries are represented with a compliance $C_{a}{ }^{3}$ and a resistance $R_{a}$, that is the arterial pressure $P_{a}$ obey to the equation

$$
C_{a} \frac{\mathrm{d} P_{a}}{\mathrm{~d} t}=\dot{Q}_{a}-\frac{P_{a}-P_{s}}{R_{a}}
$$

being $P_{s}$ the mean blood pressure after the main arteries and before the capillary bed of tissues (systemic pressure). The tissues are constituted by a global systemic compliance $C_{s}$ and a resistance. In the sequel we will consider three compartments with different metabolic activity, that are:

(1) the skeletal muscle compartment, with resistance $R_{s m}$ and blood flow rate $\dot{Q}_{s m}$;

(2) the splanchnic compartment, with resistance $R_{s p}$ and blood flow rate $\dot{Q}_{s p}$;

(3) a compartment representing other organs, with resistance $R_{o}$ and blood flow rate $\dot{Q}_{o}$.

Introducing the venous pressure $P_{v}$ and the venous compliance $C_{v}$, we are able to express the flow rates in each compartment

$$
\dot{Q}_{i}=\frac{P_{s}-P_{v}}{R_{i}}, \quad i \in\{s m, s p, o\} .
$$

Moreover, we have the following equation for the systemic pressure:

$$
C_{s} \frac{\mathrm{d} P_{s}}{\mathrm{~d} t}=\frac{P_{a}-P_{s}}{R_{a}}-\frac{P_{s}-P_{v}}{R_{s}},
$$

being $R_{s}$ the total systemic resistance:

$$
R_{s}=\left(\frac{1}{R_{s m}}+\frac{1}{R_{s p}}+\frac{1}{R_{o}}\right)^{-1} .
$$

By the conservation of the total mass of blood, the venous pressure $P_{v}$ is linearly dependent on the other pressures $P_{a}$ and $P_{s}$ : if $V_{0}$ and $V_{u}$ is the total and unstretched volume of blood, we have

$$
C_{v} P_{v}=V_{0}-V_{u}-C_{a} P_{a}-C_{s} P_{s} .
$$

\footnotetext{
${ }^{3}$ We refer to [11] for the description of vessel compliance: basically, we linearize the mechanical behaviour of the vessels and introduce a constant $C$ such that the volume variations of blood in the considered compartment are proportional to the pressure, that is $V=V_{u}+C P$, where $V_{u}$ is a constant called "unstretched volume".
} 
Equations $(1, \ldots, 5)$ can be recast in a set of two first-order linear ordinary differential equations, if the cardiac output $\dot{Q}_{a}(t)$ is given. As we know from the previous section, $\dot{Q}_{a}$ is related to heart rate and stroke volume by

$$
\dot{Q}_{a}=H R \mathrm{~V}_{\mathrm{s}},
$$

and both $H R$ and $V_{\mathrm{s}}$ are under the control of the sympathetic and parasympathetic activities. We assume

$$
\mathrm{V}_{\mathrm{s}}=V_{e, d i a}\left(P_{v}\right)-V_{u, v e n t}-\frac{P_{a}}{E},
$$

where $V_{e, d i a}$ is the end-diastolic ventricular volume (which is a known function of the venous pressure, [25]), $V_{u, v e n t}$ is the unstretched ventricular volume, and $E$ is the ventricular elastance. Defining the heart period $T=1 / H R$, we follow $[23]$ and assume

$$
\begin{aligned}
\tau_{T} \frac{d T}{d t} & =T_{0}-T-\sigma_{T, s}\left(f_{e s}\right)+\sigma_{T, v}\left(f_{e v}\right) \\
\tau_{E} \frac{d E}{d t} & =E_{0}-E-\sigma_{E}\left(f_{e s}\right) \\
f_{e s} & =f_{e s, \infty}+\left(f_{e s, 0}-f_{e s, \infty}\right) e^{-k_{e s} f_{c s}} \\
f_{e v} & =\frac{f_{e v, 0}+f_{e v, \infty} \exp \left(\left(f_{c s}-f_{c s, 0}\right) / k_{e v}\right)}{1+\exp \left(\left(f_{c s}-f_{c s, 0}\right) / k_{e v}\right)} \\
f_{c s} & =\frac{f_{\min }+f_{\max } \exp \left(\left(\tilde{P}_{a}-P_{n}\right) / k_{a}\right)}{1+\exp \left(\left(\tilde{P}_{a}-P_{n}\right) / k_{a}\right)}
\end{aligned}
$$

where $\sigma_{T, s}, \sigma_{T, v}$ and $\sigma_{E}$ are monotone increasing functions, $P_{n}$ is the reference pressure value (for example $100 \mathrm{mmHg}), \tilde{P}_{a}$ is a zero-pole filtering of the arterial pressure,

$$
\tau_{p} \frac{\mathrm{d} \tilde{P}_{a}}{\mathrm{~d} t}=P_{a}-\tilde{P}_{a}+\tau_{z} \frac{\mathrm{d} P_{a}}{\mathrm{~d} t}
$$

and $f_{e s}, f_{e v}, f_{c s}$ are respectively the efferent sympathetic activity, efferent vagal activity and the carotid sinus firing rate. In this way the baroreflex effect on the heart rate and elastance is taken into account: if we consider a low-frequency regime, $\tilde{P}_{a} \simeq P_{a}$, so $f_{c s}$ increases with respect to the arterial pressure. Thus, if $P_{a}$ is small, $T$ is reduced and $E$ is increased, and the cardiac output increases; conversely, if $P_{a}$ is big, the effect is a diminution of the cardiac output.

If the hydrodynamic resistances of the tissues were known, the equations $(1, \ldots, 13)$ would form a closed linear dynamical system with 5 state variables $P_{a}, P_{s}, T, E, \tilde{P}_{a}$. However, the vagal activity and the chemoreflex effect influence the resistances, so that we have to consider more state variables. We model the baroreflex as in [23], that is we define for $i \in\{s m, s p, o\}$ :

$$
\tau_{\tilde{R}_{i}} \frac{\mathrm{d} \tilde{R}_{i}}{\mathrm{~d} t}=\tilde{R}_{i, 0}-\tilde{R}_{i}+\sigma_{\tilde{R}_{i}}\left(f_{e s}\right),
$$

and than we follow an approach similar to [24], defining

$$
\begin{aligned}
R_{s m} & =\frac{\tilde{R}_{s m}}{1+x_{s m}}, \\
R_{s p} & =\tilde{R}_{s p}\left(1+x_{s p}\right), \\
R_{o} & =\frac{\tilde{R}_{o}}{1+x_{o}},
\end{aligned}
$$




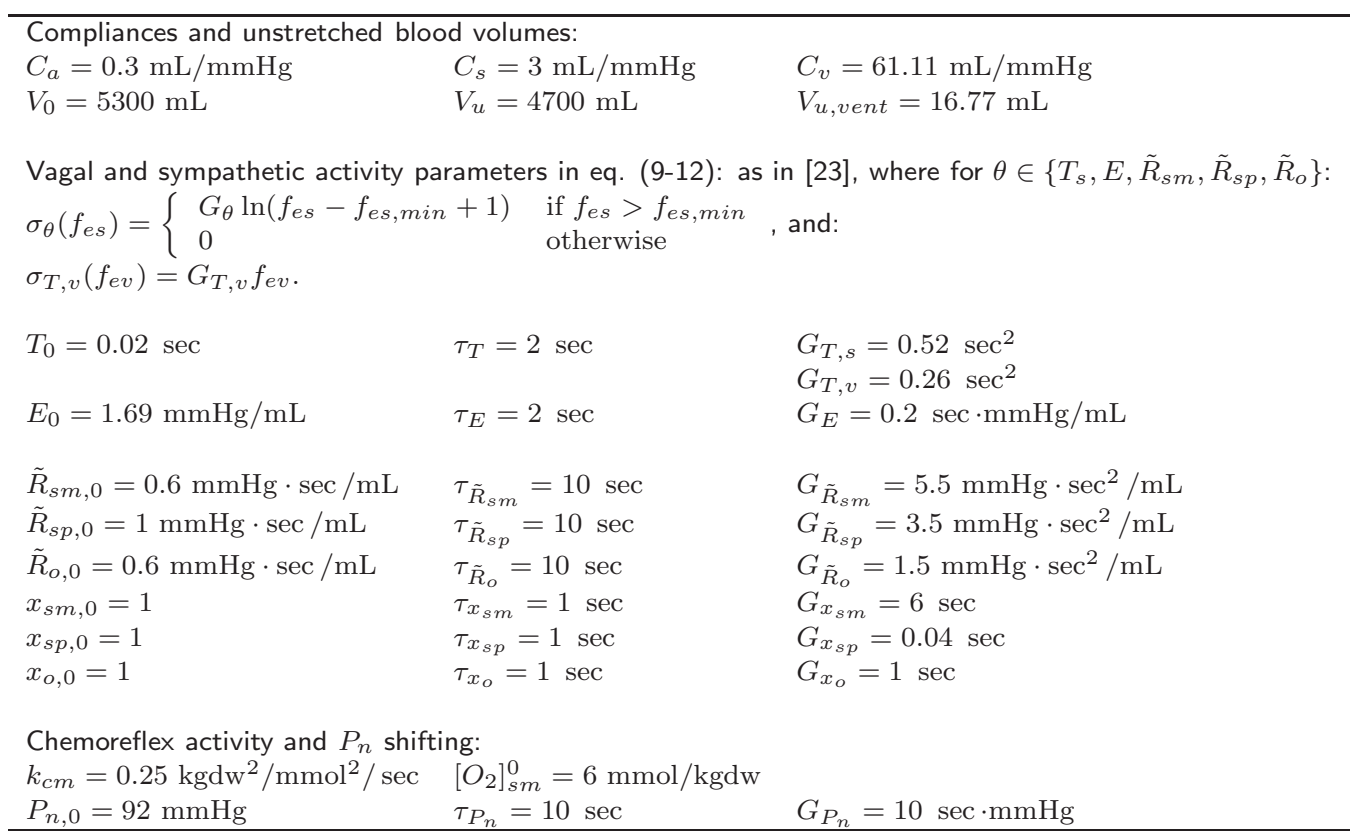

TABLE 4. Cardiovascular model parameters. Default values from [23] are used when parameters are not specified explicitly.

where $x_{i}, i \in\{s m, s p, o\}$ are new variables, that are supposed to be affected by the chemoreflex activity $f_{c m}$ :

$$
\tau_{x_{i}} \frac{\mathrm{d} x_{i}}{\mathrm{~d} t}=x_{i, 0}-x_{i}+G_{x_{i}} f_{c m}, \quad i \in\{s m, s p, o\}
$$

where $G_{x_{i}}$ are positive constants. The chemoreflex effector $f_{c m}=f_{c m}\left(\left[\mathrm{O}_{2}\right]_{s m}\right)$ is a non-increasing function of the oxygen concentration in the skeletal muscle tissue; by (16) and (15), the lower the oxygen concentration in exercising tissues, the lower their resistance and the higher the splanchnic resistance (as expected physiologically). In particular, we found a good agreement with exercise physiological data (like those of Table 2) with a quadratic dependence of $f_{c m}$ on the oxygen concentration (a compromise between the linear [24] and the exponential [16] case):

$$
f_{c m}=\left\{\begin{array}{ll}
0 & \text { if }\left[O_{2}\right]_{s m}>\left[O_{2}\right]_{s m}^{0}, \\
k_{c m}\left(\left[\mathrm{O}_{2}\right]_{s m}^{0}-\left[\mathrm{O}_{2}\right]_{s m}\right)^{2} & \text { otherwise }
\end{array},\right.
$$

where $\left[\mathrm{O}_{2}\right]_{s m}^{0}$ is a fixed threshold value for the oxygen concentration.

As pointed out in [18], [19], the response of baroreflex effector to the increased metabolic rate can be described by a shifting of the reference value $P_{n}$ of the sigmoid defined by (12). We included this effect in our model considering $P_{n}$ as a state variable affected by the chemoreflex:

$$
\tau_{P_{n}} \frac{\mathrm{d} P_{n}}{\mathrm{~d} t}=P_{n, 0}-P_{n}+G_{P_{n}} f_{c m}
$$

Equations $(1, \ldots, 18)$ form our final global cardiovascular model. We point out that in these equations $\left[\mathrm{O}_{2}\right]_{s m}$ is an input variable; in other words we need a tissue metabolism model (TMM) in order to provide this variable to the cardiovascular model (CVM). The CVM and TMM shall be coupled via the oxygen concentrations (TMM $\rightarrow \mathrm{CVM})$ and the flow rates $(\mathrm{CVM} \rightarrow \mathrm{TMM})$. In Table 4 the parameters for "standard" healthy individuals (rest 
heart rate $60 \mathrm{bpm}$, rest cardiac output $5 \mathrm{~L} / \mathrm{min}, \ldots$ ) are reported (undefined values are as in [23]); however, they have to be adapted when simulations are carried out for different subjects.

\section{Tissue Metabolism Model}

We will deal with two kinds of metabolism model. The first one, is a very simple model in which only oxygen is taken into account. The second one, is a more detailed model describing several chemical pathways (see section 1.3), which allows to simulate the dynamic of several metabolites, as $P C, G L, L A$ and so on.

\subsection{Transport and reaction of chemicals in blood and tissues}

The concentration of any chemical in the venous blood is strongly related to the concentration of that chemical in the upstream tissue. As in [2], [3], we will assume a linear relation between venous and tissue concentration, that is for any substance $C$ we assume

$$
[C]_{v, i}=\sigma_{i}^{C}[C]_{i}
$$

being $[C]_{v, i}$ the venous concentration downstream of the $i$-th tissue, $[C]_{i}$ the concentration in the tissue, and $\sigma_{i}^{C}$ the partition coefficient of the chemical $C$. The mean venous concentration $[C]_{v}$ in blood is defined as

$$
[C]_{v}=\sum_{i \in\{s m, s p, o\}} \frac{\dot{Q}_{i}}{\dot{Q}_{a}}[C]_{v, i} .
$$

As previously discussed, we assume that the only chemical species affected by the pulmonary circulation are $O_{2}$ and $\mathrm{CO}_{2}$; according to Table 3, their arterial concentrations are constant. For the other chemicals, we assume

$$
[C]_{a}=[C]_{v}, \quad C \notin\left\{\mathrm{O}_{2}, \mathrm{CO}_{2}\right\} .
$$

If the rate of oxygen consumption in a certain tissue is known, we can assume the following simple equation for the dynamic of the oxygen concentration:

$$
V_{i} \frac{\mathrm{d}\left[O_{2}\right]_{i}}{\mathrm{~d} t}=-\mathrm{OP}_{i}(t)+\dot{Q}_{i}(t)\left(\left[O_{2}\right]_{a, i}-\sigma_{i}^{O_{2}}\left[O_{2}\right]_{i}\right)
$$

where $V_{i}$ is the "volume" ${ }^{4}$ of tissue, $\left[O_{2}\right]_{i}$ is the concentration of $O_{2}$ in the tissue compartment $i, \mathrm{OP}_{i}$ is the oxygen consumption rate of the tissue (the oxygen consumed by the oxidative phosphorylation per unit time), $\dot{Q}_{i}$ is the blood flow rate in the tissue, $\left[\mathrm{O}_{2}\right]_{a, i}$ is the arterial concentration of $\mathrm{O}_{2}$, and $\sigma_{i}^{\mathrm{O}_{2}}$ is the oxygen partition coefficient. Usually the resting metabolic rate $\mathrm{OP}_{i}^{0}$ is known, as well as the resting arterial and tissue concentrations $\left[\mathrm{O}_{2}\right]_{a, i}^{0}$ and $\left[\mathrm{O}_{2}\right]_{i}^{0}$, and the mean resting flow rate $\dot{Q}_{i}^{0}$. Thus, $\sigma_{i}^{\mathrm{O}_{2}}$ can be estimated by the following balance equation at rest:

$$
\sigma_{i}^{O_{2}}=\frac{\left[O_{2}\right]_{a, i}^{0}-\mathrm{OP}_{i}^{0} / \dot{Q}_{i}^{0}}{\left[O_{2}\right]_{i}^{0}}
$$

Another way to estimate the partition coefficient is to set directly

$$
\sigma_{i}^{O_{2}}=\left[O_{2}\right]_{v, i}^{0} /\left[O_{2}\right]_{i}^{0},
$$

where $\left[\mathrm{O}_{2}\right]_{v, i}^{0}$ is the resting venous oxygen concentration: the two approaches have to give almost the same results.

\footnotetext{
${ }^{4}$ Very often, being the available physiological data for $\left[O_{2}\right]_{i}$ expressed in $\mathrm{mmol} \mathrm{kg}{ }^{-1}$ or $\mathrm{mmol} \mathrm{kgdw}{ }^{-1}$ (where $\mathrm{kgdw}$ means "kg of dry tissue") $V_{i}$ is not a volume but a weight (or a dry weight).
} 


\subsection{General multi-chemical model}

In order to take into account the interaction between several chemicals and substrates in tissues, and to obtain a deeper description of the bioenergetics of the skeletal muscle, we have to consider more complex multichemical models of the metabolism based on the main pathways described in section 1.3. In metabolic control, the chemical pathways are represented by associating a reaction rate $\psi_{R}$ to each reaction $R$. A reaction rate is a function of the chemical concentrations (and of time) which represents the molar consumption of a reference substrate in $R$ per unit time $\left(\mathrm{mmol} \mathrm{sec}^{-1}\right)$. Being each reaction $R$ referred to a certain reactant $(P C \mathrm{~b}: P C$, $P C$ s : $C R, G L \mathrm{~b}: G L, \ldots, \mathrm{MR}: A T P)$, we define $\psi_{R}$ as the molar consumption rate of that chemical.

We point out that the model has to respect the stoichiometry of the chemical reactions: for example, in the pyruvate oxidation $P Y \mathrm{o}$, if $\psi_{P Y \mathrm{o}}$ is the rate (measured for instance in mmol of $P Y \mathrm{sec}^{-1}$ ) at which $P Y$ is consumed by this reaction, we have that $5 \psi_{P Y \text { o }}$ is the corresponding rate for $N A D H$ (because 5 molecules of $N A D H$ are obtained from one of $P Y)$.

For each compartment $i$, we define a state vector $\mathbf{c}_{i}$ containing all the chemical concentration we are interested in, and a reaction rate vector $\boldsymbol{\psi}_{i}$ containing the reaction rates for each pathway:

$$
\mathbf{c}_{i}=\left(\begin{array}{c}
{[P C]_{i}} \\
{[G L]_{i}} \\
{[P Y]_{i}} \\
{[L A]_{i}} \\
{[G Y]_{i}} \\
{[F A]_{i}} \\
{[A D P]_{i}} \\
{[N A D H]_{i}} \\
{\left[O_{2}\right]_{i}} \\
{\left[C O_{2}\right]_{i}}
\end{array}\right), \quad \boldsymbol{\psi}_{i}=\left(\begin{array}{c}
P C \mathrm{~b}_{i} \\
P C \mathrm{~s}_{i} \\
G L \mathrm{~b}_{i} \\
G L \mathrm{~s}_{i} \\
G Y \mathrm{~s}_{i} \\
G Y \mathrm{~b}_{i} \\
P Y \mathrm{r}_{i} \\
L A \mathrm{o}_{i} \\
P Y \mathrm{o}_{i} \\
\mathrm{OP}_{i} \\
F A \mathrm{o}_{i} \\
\mathrm{MR}_{i}
\end{array}\right)
$$

We omitted $[C R]_{i},[A T P]_{i}$ and $[N A D]_{i}$ in the vector state because the sums $[C R]_{i}+[P C]_{i},[A T P]_{i}+[A D P]_{i}$ and $[N A D]_{i}+[N A D H]_{i}$ are conserved.

If we describe the stoichiometric relations by means of the stoichiometric matrix $\mathbf{A}$ (see Table 5), and we impose the conservation of the mass of each chemical, we obtain the following system of (nonlinear) differential equations:

$$
V_{i} \frac{\mathrm{d} \mathbf{c}_{i}}{\mathrm{~d} t}=\mathbf{A} \psi_{i}\left(\mathbf{c}_{i}, t\right)+\mathbf{b}_{i}\left(\mathbf{c}_{i}, \mathbf{c}_{a, i}, \dot{Q}_{i}, t\right),
$$

where $V_{i}$ is the tissue mass of the $i$-th compartment, $\mathbf{c}_{a, i}$ is the vector of the arterial concentrations in the incoming blood flow, and $\mathbf{b}_{i}\left(\mathbf{c}_{i}, \mathbf{c}_{a, i}, \dot{Q}_{i}, t\right)$ is the transport term. This is a quite general paradigm for a metabolism model, where the state variables are the chemical concentrations in the tissue, and the arterial concentrations and the flow rate play the role of input variables. For instance, it is possible [2] to define $\mathbf{b}_{\mathbf{i}}$ as in (19) by

$$
\mathbf{b}_{i}\left(\mathbf{c}_{i}, \mathbf{c}_{a, i}, \dot{Q}_{i}, t\right)=\dot{Q}_{i}\left(\mathbf{c}_{a, i}-\boldsymbol{\sigma}_{i} \cdot \mathbf{c}_{i}\right)
$$

being $\dot{Q}_{i}$ the flow rate in the $i$-th compartment, and $\boldsymbol{\sigma}_{i}=\left(\sigma_{i}^{P C}, \ldots, \sigma_{i}^{C 0_{2}}\right)^{T}$ the vector of the partition coefficient for each chemical $\left(\boldsymbol{\sigma}_{i} \cdot \mathbf{c}_{i}\right.$ is the component-wise vector product). We point out that $\boldsymbol{\psi}_{i}$ is a function of the state vector and of the time; the explicit expression will be given in a different way for each compartment $(i=s m, s p, o)$. In Table 5 the expression of the stoichiometric matrix and transport vector are shown: notice that some chemicals are not transported (namely $P C, G Y, A D P, N A D H$ ) and then their arterial concentration and partition coefficient are both zero. Each equation of system $(21)$ is presented in Table 6 , where $b_{P C}, \ldots, b_{C O_{2}}$ denote the components of $\mathbf{b}$. 


$$
\mathbf{A}=\left[\begin{array}{ccccccccccc}
-1 & 1 & 0 & 0 & 0 & 0 & 0 & 0 & 0 & 0 & 0 \\
0 & 0 & -1 & 1 & -1 & 10 & 0 & 0 & 0 & 0 & 0 \\
0 & 0 & 2 & -2 & 0 & 0 & -1 & 1 & -1 & 0 & 0 \\
0 & 0 & 0 & 0 & 0 & 0 & 1 & -1 & 0 & 0 & 0 \\
0 & 0 & 0 & 0 & 1 / m & -1 & 0 & 0 & 0 & 0 & 0 \\
0 & 0 & 0 & 0 & 0 & 0 & 0 & 0 & 0 & 0 & -1 \\
-1 & 1 & -2 & 2 & 1 / m & -1 & 0 & 0 & 0 & -6 & 0 \\
0 & 0 & 2 & 2 & 0 & 0 & -1 & 1 & 5 & -2 & 43 \\
0 & 0 & 0 & 0 & 0 & 0 & 0 & 0 & 0 & -1 & 0 \\
0 & 0 & 0 & 0 & 0 & 0 & 0 & 0 & 3 & 0 & 16
\end{array}\right], \quad \mathbf{b}_{i}=\dot{Q}_{i}\left[\begin{array}{c}
0 \\
{[G L]_{a}-\sigma_{i}^{G L}[G L]_{i}} \\
{[P Y]_{a}-\sigma_{i}^{P Y}[P Y]_{i}} \\
{[L A]_{a}-\sigma_{i}^{L A}[L A]_{i}} \\
0 \\
{[F A]_{a}-\sigma_{i}^{F A}[F A]_{i}} \\
0 \\
0 \\
{\left[O_{2}\right]_{a}-\sigma_{i}^{O_{2}}\left[O_{2}\right]_{i}} \\
{\left[C O_{2}\right]_{a}-\sigma_{i}^{C O_{2}}\left[C O_{2}\right]_{i}}
\end{array}\right]
$$

TABle 5. Stoichiometric matrix $\mathbf{A}$ and transport vector $\mathbf{b}_{i}$.

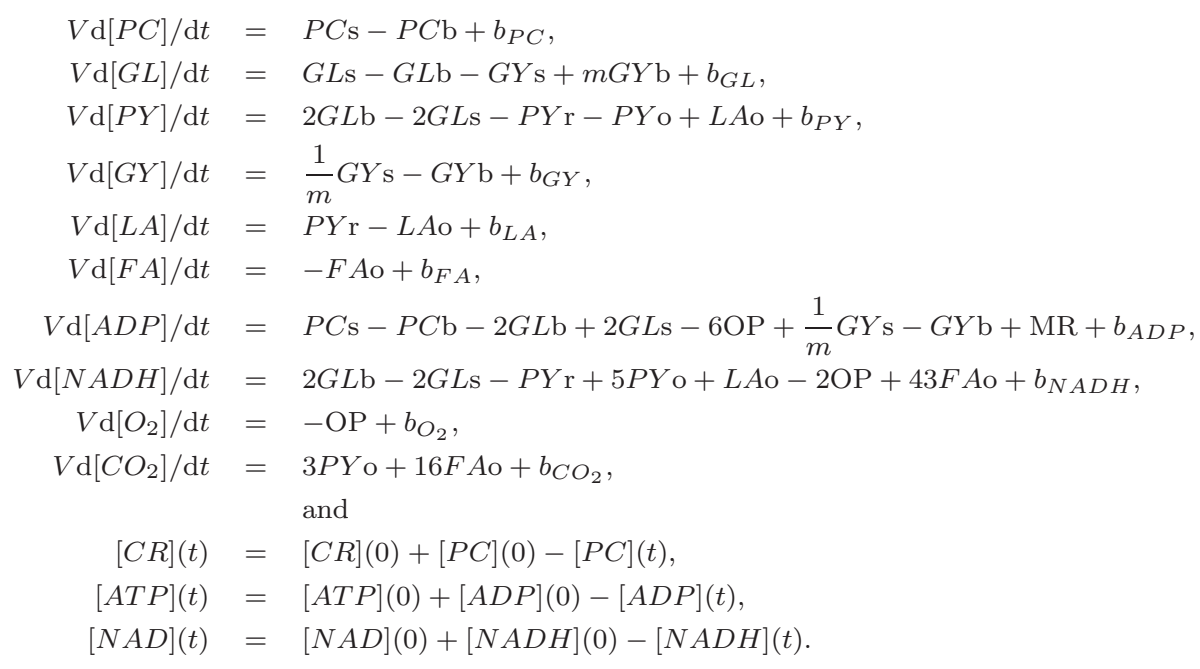

TABLE 6. Metabolism kinetics (we omitted the subscripts).

\subsubsection{Skeletal muscle compartment}

As in [2] we assume that in skeletal muscle $(i=s m)$ Michaelis-Menten laws hold for the reaction rates. These reaction rates are controlled by the phosphorylation state $P S$ and the redox state $R S$, where

$$
P S=\frac{[A D P]}{[A T P]}, \quad R S=\frac{[N A D H]}{[N A D]} .
$$

The main idea [2] is that the higher $P S$, the higher the reaction rate of the $A T P$ producing pathways; analogously, the lower $R S$, the higher the reaction rate of the $N A D H$ producing pathways. The complete set of rates is presented in Table 7: moreover we assume $G L \mathrm{~s}_{s m}=0$ as discussed in section 1.3.

In the Michaelis-Menten laws there are parameters which are not provided by experiments. In fact, in each reaction $R$ we have a maximal rate coefficient $\phi_{R}$ and one or more characteristic constants $k_{R}$ : these coefficient 
are not known a priori, except in a few cases. Thus, parameter identification is mandatory in order to find the coefficients that reproduce the dynamic of the metabolic pathways fitting the available physiological data.

\begin{tabular}{|c|c|c|}
\hline Reaction & Stoichiometry & Rate \\
\hline$P C$ s & $C R+A T P \longrightarrow P C+A D P$ & $\phi_{P C s} \frac{1}{P S / k_{P C \mathrm{~s}}+1}[C R]$ \\
\hline$P C \mathrm{~b}$ & $P C+A D P \longrightarrow C R+A T P$ & $\phi_{P C b} \frac{P S}{P S+k_{P C \mathrm{~b}}}[P C]$ \\
\hline$G L \mathrm{~b}$ & $G L+2 A D P+2 N A D \longrightarrow 2 P Y+2 A T P+2 N A D H$ & $\phi_{G L b} \frac{P S}{P S+k_{G L \mathrm{~b}}^{P S}} \frac{1}{R S / k_{G L \mathrm{~b}}^{R S}+1}[G L]$ \\
\hline$G Y \mathrm{~s}$ & $m G L+A T P \longrightarrow G Y+A D P$ & $\phi_{G Y s} \frac{1}{P S / k_{G Y \mathrm{~s}}+1}[G L]$ \\
\hline$G Y \mathrm{~b}$ & $G Y+A D P \longrightarrow m G L+A T P$ & $\phi_{G Y b} \frac{P S}{P S+k_{G Y \mathrm{~b}}}[G Y]$ \\
\hline$P Y \mathrm{r}$ & $P Y+N A D H \longrightarrow L A+N A D$ & $\phi_{P Y r} \frac{R S}{R S / k_{P Y \mathrm{r}}+1}[P Y]$ \\
\hline$L A$ o & $L A+N A D \longrightarrow P Y+N A D H$ & $\phi_{L A o} \frac{1}{R S / k_{L A o}+1}[L A]$ \\
\hline$P Y \mathrm{o}$ & $P Y+5 N A D \longrightarrow 5 N A D H+3 C_{2}$ & $\phi_{P Y o} \frac{1}{R S / k_{P Y o}+1}[P Y]$ \\
\hline OP & $3 A D P+N A D H+\frac{1}{2} \mathrm{O}_{2} \longrightarrow 3 A T P+N A D+\mathrm{H}_{2} \mathrm{O}$ & $V_{\mathrm{O}_{2}}^{\max } \frac{R S}{R S+k_{\mathrm{OP}}} \frac{1}{1+\left(\frac{K_{A D P}}{[A D P]}\right)^{n}}\left[O_{2}\right]$ \\
\hline$F A$ o & $F A+43 N A D \longrightarrow 16 C O_{2}+43 N A D H$ & $\phi_{F A o} \frac{1}{R S / k_{F A o}+1}[F A]$ \\
\hline MR & $A T P \longrightarrow A D P+$ energy & $M R(t)$ \\
\hline
\end{tabular}

Phosphorylation state: $P S=[A D P] /[A T P]$
Redox state: $R S=[N A D H] /[N A D]$

TABLE 7. Reaction rates for the skeletal muscles (the subscript $i=s m$ is omitted).

\subsubsection{Splanchnic compartment}

Splanchnic organs, as liver, provide glucose and free fatty acids if the body needs them. They may also convert pyruvate in glucose $(G L \mathrm{~s} \neq 0)$. As metabolic rate in these organs is not that high, we consider as state variables only $G L, G Y, P Y, L A, F A, \mathrm{O}_{2}$ and $\mathrm{CO}_{2}$. Moreover, we assume simple linear reaction rates, with the exception of the oxidative phosphorylation: the expressions are listed in Table 8. As for the skeletal muscle tissue, the coefficients of these expressions have to be (at least partially) estimated by means of parameter identification techniques.

\subsubsection{Other organs}

In the third compartment $i=o$ representing the remaining tissues, we assume that the metabolism is known. The venous concentration of the transported chemicals is set to the constant standard value for the considered substrate. 


\begin{tabular}{ccc}
\hline Reaction & Stoichiometry & Rate \\
\hline$G L \mathrm{~b}$ & $G L \longrightarrow 2 P Y$ & $\phi_{G L b}[G L]$ \\
$G L \mathrm{~s}$ & $2 P Y \longrightarrow G L$ & $\phi_{G L b}[P Y]$ \\
$G Y \mathrm{~s}$ & $m G L \longrightarrow G Y$ & $\phi_{G Y s}[G L]$ \\
$G Y \mathrm{~b}$ & $G Y \longrightarrow m G L$ & $\phi_{G Y b}[G Y]$ \\
$P Y \mathrm{r}$ & $P Y \longrightarrow L A$ & $\phi_{P Y r}[P Y]$ \\
$L A \mathrm{o}$ & $L A \longrightarrow P Y$ & $\phi_{L A o}[L A]$ \\
$P Y \mathrm{o}$ & $P Y \longrightarrow 3 C O_{2}$ & $\phi_{P Y o}[P Y]$ \\
$F A \mathrm{o}$ & $F A \longrightarrow 16 C O_{2}$ & $\phi_{F A o}[F A]$
\end{tabular}

TABLE 8. Reaction rates for the splanchnic organs (the subscript $i=s p$ is omitted).

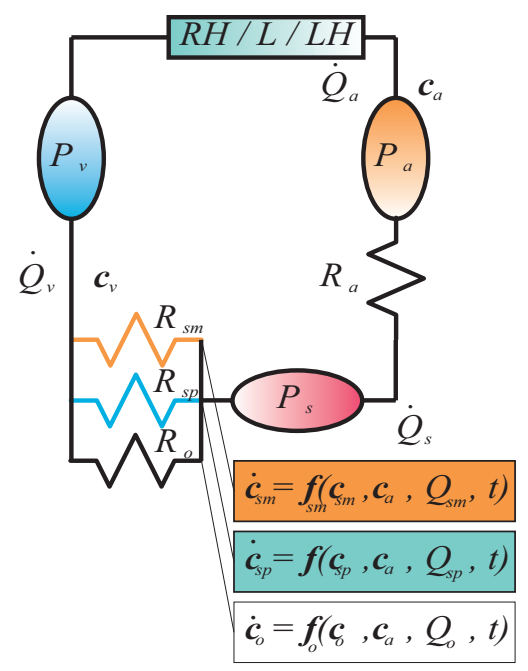

Figure 3. Coupling between the cardiovascular system and the metabolism.

\section{Coupled Cardiovascular - Metabolism models: PARAmeter estimation}

The cardiovascular and the metabolism models described in the previous sections can be coupled in order to describe the mutual interaction between hemodynamics and bioenergetics (see fig. 3).

This coupling is due to the dependence $(15,16,17)$ of the hydraulic resistance $R_{i}, i \in\{s m, s p, o\}$, on the oxygen concentration $\left[\mathrm{O}_{2}\right]_{s m}$ in the $s m$ tissue compartment, which is the simplest case of chemoreflex effect. More generally, we could assume a dependence on other chemicals. The global model is intended to give a prediction of the cardiovascular and metabolic behaviour under effort conditions; the main input variable is the skeletal muscle metabolic rate $\mathrm{MR}_{i}(t)$, which represents the rate of conversion of $A T P$ in $A D P$, proportional to the exercise workload. As output variables, we obtain the cardiac output, heart rate, local blood flow and chemical concentration of several substrates in the blood. 
However, as we pointed out previously, we have to estimate the unknown parameters of the Michaelis-Menten laws for the reaction rates (see [4] for a systematic approach to parameter identification in metabolic systems). Let denote by $\mathbf{p}$ the vector of these parameters: the idea is to minimize a cost functional $J=J(\mathbf{p})$ which measures the "distance" between a set of control clinic data $\mathbf{y}_{o b}=\mathbf{y}_{o b}(t)$ and the corresponding quantities $\mathbf{y}$ predicted by the model. Formally, we can set

$$
J(\mathbf{p})=\left\|\mathbf{y}(\mathbf{p}, t)-\mathbf{y}_{o b}(t)\right\|^{2},
$$

where $\|\cdot\|$ is a suitable norm, as the 2 -norm $\|\mathbf{f}\|_{2}=\sqrt{\int \mathbf{f}(t) \cdot \mathbf{f}(t) \mathrm{d} t}$ or the $\infty$-norm $\|\mathbf{f}\|_{\infty}=\max _{t, i}\left|f_{i}(t)\right|$, and $\mathbf{y}(\mathbf{p}, t)$ is the vector of predicted quantities with the set of parameters $\mathbf{p}$. Each evaluation of the cost function $J$ requires to solve the model, that is to find the solution of a system of differential equations. The goal is to obtain (a suitable approximation of) the optimal vector $\mathbf{p}^{*}$ in the set $P_{a d}$ of admissible parameters:

$$
\text { find } \mathbf{p}^{*}: \quad J\left(\mathbf{p}^{*}\right)=\min _{\mathbf{p} \in P_{a d}} J(\mathbf{p}) \text {. }
$$

The set $P_{a d}$ has to represent all the constrains on the parameters; for instance, every component of $\mathbf{p} \in P_{a d}$ has to be greater than zero.

Problem (23) is an inverse problem, expressed in the form of an optimal control problem. We used both standard line-search (see for instance [20]) and genetic algorithms [5] to identify the best parameters: the observed variables were the basal tissue concentration as in [2]. In particular, we found that genetic algorithms allow to achieve a better identification due to their global behaviour; the drawback is obviously an augmented computational time with respect to the so-called "deterministic methods". The Matlab Optimization toolbox (line-search algorithms) and the GAOT library [10] (genetic algorithms) have been used.

\section{Model Simulations}

Here we present some preliminary results obtained simulating the coupling between the extended metabolism model described in section 3.2 and the cardiovascular model (section 2). We used a low-order Runge-Kutta scheme for stiff systems to solve the model equations.

We considered a 10 min incremental exercise, with a linear increasing workload: the metabolic rate $\mathrm{MR}_{s m}(t)$ of the skeletal muscle compartment (consumption of ATP per unit time) was increased starting from the basal value $\mathrm{MR}_{s m}^{0}(t=0)$ up to $30 \mathrm{MR}_{s m}^{0}(t=10 \mathrm{~min})$ and then again set equal to $\mathrm{MR}_{s m}^{0}$ (rest). Being the energy expenditure of the whole body basal metabolism approximately equal to $75 \mathrm{~W}$, and the skeletal muscle rest blood flow $\dot{Q}_{s m}^{0} \simeq \dot{Q}_{a}^{0} / 5$, we can estimate the rest skeletal muscle power to be $15 \mathrm{~W}$. Therefore, if the power output was proportional to MR, the peak power of the exercise simulation would be about $450 \mathrm{~W}$; actually, the ATP breakdown become less and less efficient as the workload increases, so that the (power output)/MR ratio is not a constant, but rather a decreasing function of MR. If we assume that under high effort condition the metabolic efficiency is reduced by $35 \%$ [3,13], then we can estimate a $300 \mathrm{~W}$ peak power output.

The evolution in time of the main hemodynamical variables is shown in Figure 4 . The time course of the vascular resistances, for instance, shows that during exercise the skeletal muscle becomes the most blood demanding compartment; this fact perturbs the overall systemic resistance, which in turn causes the increase of heart rates (and stroke volume, not shown here) due to the baroreflex effector. Moreover, the shifting of the reference value for the pressure in the carotid sinus sigmoid curve causes an increase of the mean arterial pressure.

In Figure 5 a few relevant venous chemical concentrations are shown $\left(\mathrm{O}_{2}, \mathrm{CO}_{2}, L A\right.$ and $\left.P Y\right)$. As expected, a reduction in the venous $\mathrm{O}_{2}$ content is observed; moreover the high level of skeletal muscle metabolic rate causes an increase in carbon dioxide (6.5\%), and a significant accumulation of lactate (900\%) and pyruvate (1000\%). Increments in lactate level of the same order are found in the ramp tests described in [6]; however, our results are to be considered as preliminary investigations in a very complex field, rather than exhaustive numerical simulations of the hemodynamics and biochemistry of an exercise. 

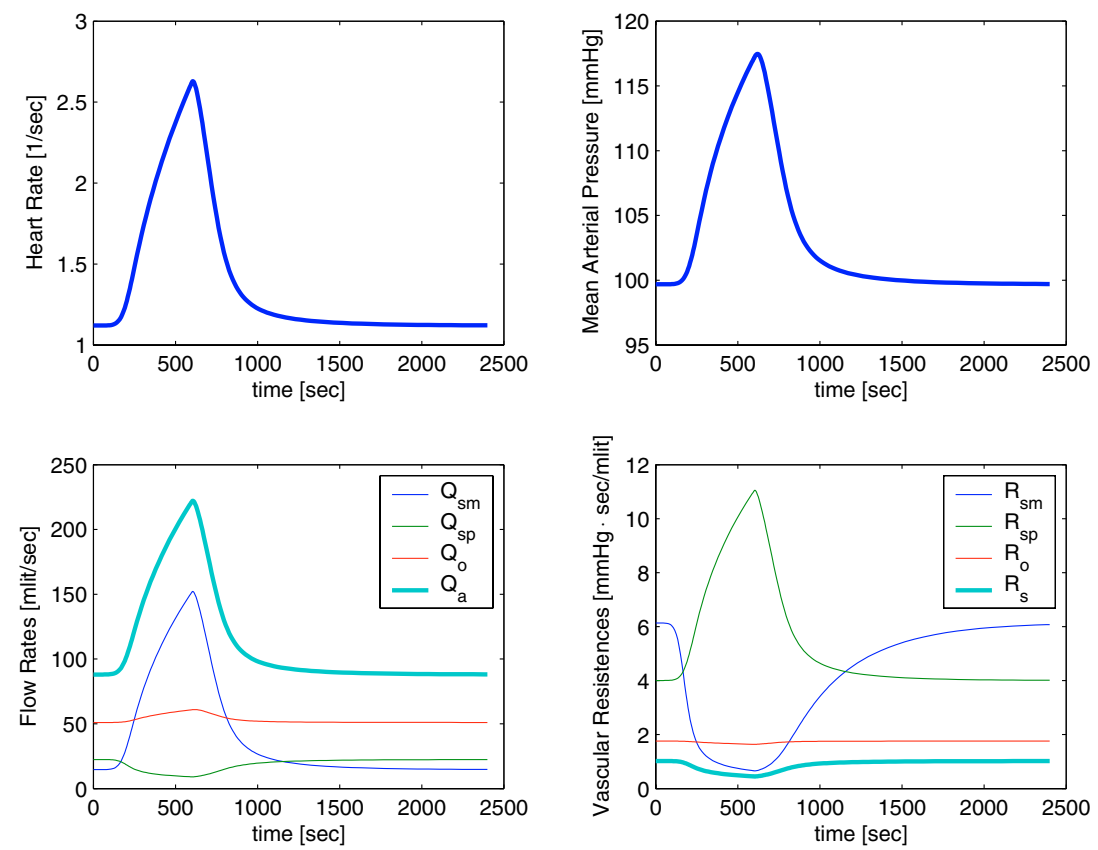

Figure 4. Heart rates, mean arterial pressure, flow rates and vascular resistances in a 10 minutes incremental exercise.
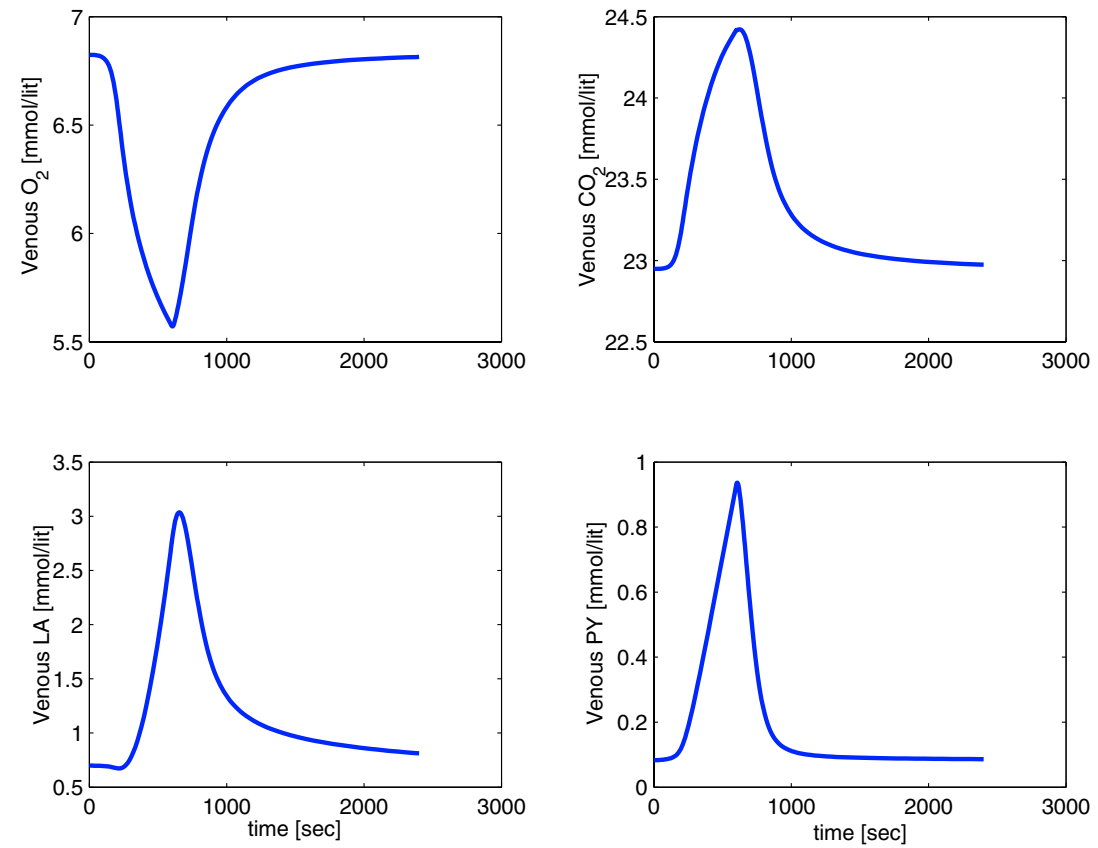

FiguRE 5. Venous concentrations of oxygen, carbon dioxide, lactate and pyruvate in a 10 minutes incremental exercise. 


\section{REFERENCES}

[1] G.A. Brooks, T.D. Fahey, and T.P. White. Human Bioenergetics and Its Applications. Mayfield Publishing Company, 1987.

[2] M.E. Cabrera, G.M. Saidel, and S.C. Kalhan. Role of $\mathrm{O}_{2}$ in regulation of lactate dynamics during hypoxia: Mathematical model and analysis. Ann. of Biomed. Eng., 26:1-27, 1998.

[3] M.E. Cabrera, G.M. Saidel, and S.C. Kalhan. Lactate metabolism during exercice: analysis by an integrative systems model. Am. J. Physiol., 277:1522-1536, 1999.

[4] E. Carson, C. Cobelli, and L. Finklestein. The mathematical modeling of metabolic and endocrine systems. Wiley, 1983.

[5] L. Davis. The Handbook of Genetic Algorithmms. Van Nostrand Reingold, New York, 1991.

[6] H.J. Green, R.L. Hughson, G.W. Orr, and D.A. Ranney. Anaerobic threshold, blood lactate, and muscle metabolites in progressive exercise. J. Appl. Physiol., 54(5):1032-1038, 1983.

[7] A.C. Guyton. Textbook of Medical Physiology. W.B. Saunders Company, Philadelphia, 1981.

[8] J. Hansen, M. Sander, and G.D. Thomas. Metabolic modulation of sympathetic vasoconstriction in exercising skeletal muscle. Acta Physiol. Scand., 168:489-503, 2000.

[9] M. Hargreaves. Skeletal muscle metabolism during exercise in humans. Clinical and Experimental Pharm. and Physiol., 27:225$228,2000$.

[10] C. Houck and J. Joines. A genetic algorithm for function optimization: A matlab implementation. technical paper ncsu-ie tr 95-09, NC State University, 1995.

[11] J. Keener and J. Sneyd. Mathematical Physiology. Springer, New York, 1998.

[12] B. Korzeniewski and J.A. Zoladz. A model of oxidative phosphorylation in mammalian skeletal muscle. Biochem. Chem., 92:17-34, 2001.

[13] P. Krustrup, R.A. Ferguson, M. Kjaer, and J. Bangsbo. ATP and heat production in human skeletal muscle during dynamic exercise: higher efficiency of anaerobic than aerobic ATP resynthesis. J Physiol., 549(Pt. 1):1255-269, 2003.

[14] M.J. Kushmerick. Energy balance in muscle activity: Simulation of ATPase coupled to oxidative phosphorylation and to creatine kinase. Comparative Biochem. and Physiol., 120:109-123, 1998.

[15] M.J. Lambeth and M.J. Kushmerick. A computational model for glycogenolysis in skeletal muscle. Ann Biomed Eng., 30:808$827,2002$.

[16] K. Lu, J.W. Clark J.R., F.H. Ghorbel, D.L. Ware, J.B. Zwischenberg, and A. Bidani. Whole-body gas exchange in human predicted by a cardiopulmonary model. Cardiov. Eng., 3:1-19, 2002.

[17] E. Magosso, A. Feliciani, and M. Ursino. A mathematical model of cardiovascular response to dynamic exercise. In Proceedings of the $23^{\text {rd }}$ Annual Conference of the IEEE Engineering in Medicine and Biology Society, 2001.

[18] Y. Papelier, P. Escourrou, J.P. Gauthier, and L.B. Rowell. Carotid baroreflex control of blood pressure and heart rate in men during dynamic exercise. J. Appl. Physiol., 77:502-506, 1994.

[19] Y. Papelier, P. Escourrou, F. Helloco, and L.B. Rowell. Muscle chemoreflex alters carotid sinus baroreflex response in humans. J. Appl. Physiol., 82:577-583, 1997.

[20] A. Quarteroni, R. Sacco, and F. Saleri. Numerical Mathematics. Springer, New York, 2000.

[21] R.S. Richardson, L.J. Haseler, A.T. Nygren, S. Bluml, and L.R. Frank. Local perfusion and metabolic demand during exercise: a noninvasive MRI method of assessment. J. Appl. Physiol., 91(4):1845-1853, 2001.

[22] B. Saltin. Capacity of blood flow delivery to exercising skeletal muscle in humans. Am. J. Card., 62:30E-35E, 1988.

[23] M. Ursino. Interaction between carotid baroregulation and the pulsating heart: a mathematical model. Am. J. Physiol. (Heart Circ. Physiol.), 275:H1733-H1747, 1998.

[24] M. Ursino, E. Magosso, and G. Avanzolini. A mathematical model of $\mathrm{CO}_{2}$ effect on cardiovascular regulation. Am. J. Physiol. (Heart Circ. Physiol.), 281:H2036-H2052, 2001.

[25] H.G. Waistra. Modelonderzoek aan de baroreflex bloeddrunkregelin. Internal report, TU Eindhoven, 1981. 